\title{
Predictability of the spontaneous lumbar curve correction after selective thoracic fusion in idiopathic scoliosis
}

\author{
Rob C. Jansen · Lodewijk W. van Rhijn • \\ Eric Duinkerke · André van Ooij
}

Received: 19 June 2006/Revised: 15 December 2006/ Accepted: 14 January 2007/Published online: 9 February 2007

(C) Springer-Verlag 2007

\begin{abstract}
In this study we tried to achieve a better understanding of the biodynamic mechanism of balance in the scoliotic spine. Therefore we focused on the pre- and postoperative spine of patients with idiopathic scoliosis with a primary thoracic curve and a secondary lumbar curve. Several studies showed that the lumbar curve spontaneously corrects and improves after selective thoracic fusion. We try to understand and describe this spontaneous compensatory lumbar curve correction after selective thoracic correction and fusion. We performed a retrospective examination of pre- and postoperative radiographs of the spine of 38 patients with idiopathic scoliosis King type II and III. Frontal Cobb angles of the thoracic and lumbar curves were assessed on pre- and postoperative antero-posterior and side bending radiographs. We determined the postoperative corrections of the thoracic and lumbar curves. Relative (\%) corrections and correlations of the postoperative corrections were calculated. The group was divided in three subgroups, depending on lumbar curve modifier, according to Lenkes classification system. The calculations were done for the whole group as for each subgroup. As expected, significant correlations were present between the relative correction of the main thoracic and the lumbar curve (mean $R=0.590 ; P=0.001)$. The relation between relative thoracic and lumbar correction decreased with the lumbar modifier type. This study shows a highly sig-
\end{abstract}

R. C. Jansen $(\varangle) \cdot$ L. W. van Rhijn .

E. Duinkerke · A. van Ooij

Department of Orthopaedic Surgery,

Academic Hospital Maastricht, PO Box 5800,

6202 AZ Maastricht, The Netherlands

e-mail: robjansen_80@yahoo.com nificant correlation between the relative corrections of the main thoracic curve and the lumbar curve after selective thoracic fusion in idiopathic scoliosis. This correlation depends on lumbar curve modifier type. This new classification system seems to be of great predictable value for the spontaneous correction of the lumbar curve. Depending on the curve-type, a different technique for predicting the outcome should be used. The lumbar curve correction does not occur throughout the whole lumbar curve. Most correction is achieved in the upper part of the curve. The distal lumbar curve seems to be more rigid and less important in the spontaneous curve correction.

Keywords Idiopathic scoliosis - Surgical correction Selective thoracic fusion - Spontaneous correction . Biomechanics

\section{Introduction}

Selective thoracic fusion is an accepted technique for adolescent idiopathic scoliosis King type II. Several studies showed that the lumbar curve can spontaneously correct and improve after selective thoracic fusion $[1,3,6,7,10,11,15,16]$. We were interested in how the lumbar curve corrects following selective thoracic fusion and whether one can explain or predict the behaviour of the lumbar curve. In the literature it is said that the lumbar curve spontaneously corrects to balance the thoracic curve after selective thoracic fusion $[3,6,7,10,16]$. Most authors assumed a mechanism whereby improvement of the lumbar curve occurred through counterbalancing the surgical correction of the thoracic curve. The correction of the 
lumbar curve is said to echo the correction obtained for the thoracic curve. Because of these hypotheses we postulate there should be a correlation between the correction of the lumbar and thoracic curves of a scoliosis.

In an earlier study about the behaviour of the lumbar curve after selective thoracic fusion we found as expected, a significant correction of the lumbar curve following selective thoracic fusion for adolescent idiopathic scoliosis King type II [17]. These results suggested that the lumbar curve spontaneously followed the thoracic curve, but we did not find a significant correlation between these corrections of the thoracic and lumbar curve [17]. So, the correction of the lumbar curve was not a reflection of the thoracic correction.

Nowadays we use third generation operation techniques (segmental hook-rod system) and new classification systems are introduced. Recently Lenke et al. [12] developed a new classification system in which he divided the lumbar curve in three subgroups: lumbar modifier A, B and C. The selection of specific operative treatments depends on this modifier. In thoracic scoliosis with a lumbar modifier $\mathrm{C}$ it sometimes seems to be more appropriate to fuse the lumbar curve as well [12]. So, it is more likely to find a more significant correlation between the lumbar and thoracic curve correction for type A and type B then for type C.

It would be interesting to know whether in thoracic scoliosis with a lumbar modifier A, B or C the significance of the relationships between the curves varies with the modifier type. Also, postoperatively, one could use the quantitative description of the relative correction of the lumbar curve, to assess whether, in the individual patient, this new classification system can be used as a correct predictor of outcome results. This should be investigated for all three-modifier types. The higher the correlation coefficient between the relative corrections of thoracic and lumbar curves, the higher the predictability of the correction of the unfused lumbar curve.

To get more insight in how the lumbar curve corrects after thoracic fusion, we did a study on patients with a thoracic scoliosis who had been operated on using a selective thoracic fusion.

\section{Materials and methods}

All cases of idiopathic scoliosis, which underwent selective thoracic fusion in the Academic Hospital of Maastricht (AZM) between 1986 and 2000 were re- viewed. Inclusion criteria were: idiopathic scoliosis with a primary right thoracic curve pattern, curves classified as King type II or III [7], selective thoracic fusion using third generation instrumentation, the lowest instrumented vertebra not beyond L1, a complete set of pre- and postoperative radiographs. Initially 44 patients were included. Six patients were lost to follow up; one because of moving abroad and five others because of an incomplete set of radiographs at follow up. A total of 38 patients, 9 male, and 29 female, were treated by selective thoracic fusion using third generation instrumentation. We used a posterior double rod instrumentation. For fixation we used hooks at the cranial end and mostly hooks and sometimes screws at the caudal end. The mean age at surgery was 17 years (13-36).

For radiographic evaluation we used the standing antero-posterior and lateral radiographs and supine bending radiographs of the spine. We assessed the frontal Cobb angles of the proximal thoracic (PT), main thoracic (MT) and lumbar (L) curves and we assessed the apical vertebral translation (AVT) and rotation (perdriolle) of the curves. Trunkal shift and L4-tilt were determined. In the sagittal plane five segments were measured to determine sagittal alignment: T5-T12, T10-T12, T12-L2, L1-L5 and L4-sacrum. All measurements were done by the same investigator (third author). Radiographs were examined preoperative, 1-year postoperatively and at final follow up.

In this study, follow up was 1-year and mean final follow up was 47 months (with a range of 12-109). Because we were interested in how the lumbar curve corrects after thoracic fusion we assumed that after 1 year a stable situation is reached in the thoracic and lumbar spine. Follow up was too heterogeneous for proper statistical analysis.

Because we wanted to assess whether classification according to Lenke influenced treatment outcome, we divided the group in three subgroups according to lumbar modifier A, B and C according to Lenke [12] (Table 1).

The spontaneous decrease of the lumbar curve after selective thoracic fusion leads us to assume that the equilibrium in the scoliotic spine can be described by linear relationships between the different levels and planes of the scoliosis. To study relationships between thoracic and lumbar curves, linear correlation coefficients between the lumbar and thoracic curves were calculated.

In order to analyse whether, in the individual patient, this new classification system can be used as a correct predictor of outcome results, the correlation 
Table 1 Classification system for the lumbar spine as proposed by Lenke et al. [12]

\begin{tabular}{ll}
\hline LSM & Characteristics \\
\hline A & CSVL falls between lumbar pedicles up to stable \\
& vertebra \\
Must have thoracic apex & \\
If in doubt as to whether CSVL touches medial aspect \\
of lumbar apical pedicle, choose type B \\
Includes King type III, IV and V \\
CSVL falls between medial border of lumbar concave \\
pedicle and lateral margin of apical vertebral body of \\
bodies (if apex is a disc) \\
Must have a thoracic apex \\
If in doubt as to whether CSVL touches lateral margin \\
of apical vertebral body/-ies, choose type B \\
Includes King type II, III and V \\
CSVL falls medial to lateral aspect of lumbar apical \\
vertebral body/-ies \\
May have thoracic, thoracolumbar and/or lumbar apex \\
If in doubt as to whether CSVL touches lateral margin \\
of apical vertebral body/-ies, choose type B \\
Includes King types I, II, V, double major, triple major \\
thoracolumbar and lumbar curves
\end{tabular}

LSM lumbar spine modifier, $C S V L$ central sacral vertical line

coefficient between the relative (\%) corrections of the thoracic and lumbar curves were calculated for the whole group as for the three subgroups. The higher the correlation coefficient the higher the predictability for the correction of the unfused lumbar curve for that specific group. Relative corrections were computed for the MT and L curves in each patient using the following formula: (preoperative curve - postoperative curve)/preoperative curve $\times 100(\%)$.

The paired samples $t$-test was used for analyses between the groups. Pearson correlation analyses were used to identify linear relations between the continuous variables. Statistical analyses were performed using SPSS (V12.0, SPSS Inc., Chicago, IL). All probability $(P)$ values in this study were calculated within a confidence interval of $95 \%$.

\section{Results}

The individual values of the radiographic parameters of the 38 patients are given in Table 2. The mean values of the thoracic and lumbar curves and apical vertebral translation are given in Table 3 for both the whole group and the three subgroups. The lowest instrumented vertebra was L1 in 26 patients $(68.4 \%)$ and T12 in the remaining $12(31.6 \%)$. The apices of the main thoracic curves were most often situated at T8 $(n=13), \mathrm{T} 9(n=20)$ and T10 $(n=3)$. The apices of the lumbar curves were most often situated at L2 $(n=13)$ and L3 $(n=22)$. Values of sagittal alignment are given in Table 4 . The mean apical vertebral rotation in the main thoracic and lumbar curve were $21^{\circ}$ and $10^{\circ}$ preoperatively and $18^{\circ}$ and $10^{\circ}$ postoperatively.

To determine balance in the patients spine, we measured trunkal shift. Preoperatively, mean trunkal shift was $10 \mathrm{~mm}$ and postoperatively it was $14 \mathrm{~mm}$. This shows a minimal decrease in balance in the scoliotic spine of these patients. If we determine trunkal shift depending on lumbar modifier, it improves $2 \mathrm{~mm}$ for Lenke A curves and deteriorates $6 \mathrm{~mm}$ for Lenke B curves and $9 \mathrm{~mm}$ for Lenke $\mathrm{C}$ curves but these are not significant.

For the whole group, L4-tilt increased from $8^{\circ}$ preoperative to $9^{\circ}$ postoperative, which is just significant $(P=0.049)$. For the subgroups, the postoperative changes in L4-tilt were not significant. Lenke A: pre 4 post 5; Lenke B: pre 8 post 10; Lenke C: pre 16 post 15 .

Mean values of sagittal alignment are given in Table 4 for the entire group as for the three subgroups. Analysing these values showed no significant difference in pre- and postoperative sagittal alignment.

We found a significant correlation between the frontal Cobb angles of the main thoracic and lumbar curves preoperatively $(R=0.697 ; P<0.001), 1$ year postoperatively $(R=0.689 ; P<0.001)$ and at final follow-up $(R=0.664 ; P<0.001)$. After operation, both curves had improved significantly (mean thoracic correction $54 \%$, SD 14\%; mean lumbar correction $44 \%$, SD $21 \%)$.

In order to find a correlation between the correction of the main thoracic and lumbar curves, we used the relative (\%) corrections of the curves (Table 5). One year postoperatively, we found a weak but highly significant correlation between relative thoracic and lumbar curve correction for the whole group $(R=0.590 ; P<0.001)$ (Fig. 1$)$. The correlation coefficient between the relative correction of the thoracic and lumbar curve seems to decrease with the lumbar modifier (A, B, C) (Table 5). For lumbar modifier $\mathrm{A}$, the correlation is strongest $(R=0.698$; $P=0.004)$.

We did the same calculations for the values at final follow-up with a mean follow-up of 47 months. It showed the same decrease in correlation depending on the lumbar modifier. For the whole group, the correlation between the relative corrections was again highly significant $(R=0.530 ; P=0.001)$. The correlation was strongest for the lumbar modifier A group $(R=0.630 ; P=0.012)$. 
Table 2 Clinical details of our patients with idiopathic scoliosis

\begin{tabular}{|c|c|c|c|c|c|c|c|c|c|c|c|c|c|c|c|c|c|}
\hline \multirow[b]{3}{*}{ Patient } & \multirow[b]{3}{*}{ Lenke } & & & \multicolumn{5}{|c|}{ Vertebrae } & \multirow{2}{*}{\multicolumn{3}{|c|}{$\frac{\text { Pre-operative }}{\text { Frontal Cobb angle }}$}} & \multirow{2}{*}{\multicolumn{3}{|c|}{$\frac{\text { Bending }}{\text { Frontal Cobb angle }}$}} & \multirow{2}{*}{\multicolumn{3}{|c|}{$\frac{\text { Follow-up } 12 \text { months }}{\text { Frontal Cobb angle }}$}} \\
\hline & & \multicolumn{2}{|l|}{ Age } & \multicolumn{3}{|c|}{ Apex } & \multirow{2}{*}{\multicolumn{2}{|c|}{ Instr }} & & & & & & & & & \\
\hline & & Years & $\mathrm{M} / \mathrm{F}$ & PT & MT & $\mathrm{L}$ & & & PT & MT & $\mathrm{L}$ & PT & MT & $\mathrm{L}$ & PT & MT & $\mathrm{L}$ \\
\hline 1 & A & 28 & $\mathrm{~F}$ & $\mathrm{~T} 1$ & T9 & L3 & $\mathrm{T} 4$ & L1 & 24 & 57 & 27 & 10 & 46 & 13 & 12 & 25 & 15 \\
\hline 2 & A & 14 & $\mathrm{~F}$ & $\mathrm{~T} 1$ & $\mathrm{~T} 8$ & L2 & T3 & L1 & 24 & 65 & 30 & 19 & 58 & 3 & 18 & 35 & 18 \\
\hline 3 & A & 16 & $\mathrm{M}$ & $\mathrm{T} 4$ & $\mathrm{~T} 11$ & L4 & $\mathrm{T} 4$ & L1 & 29 & 55 & 34 & 21 & 30 & 1 & 23 & 27 & 19 \\
\hline 4 & A & 20 & $\mathrm{~F}$ & $\mathrm{~T} 3$ & $\mathrm{~T} 8$ & L1 & $\mathrm{T} 4$ & $\mathrm{~T} 12$ & 41 & 60 & 40 & 41 & 28 & 0 & 23 & 30 & 15 \\
\hline 5 & A & 18 & $\mathrm{~F}$ & $\mathrm{~T} 3$ & T9 & L4 & $\mathrm{T} 4$ & L1 & 17 & 46 & 22 & 11 & 22 & 13 & 11 & 14 & 7 \\
\hline 6 & A & 14 & $\mathrm{~F}$ & $\mathrm{~T} 2$ & $\mathrm{~T} 9$ & L3 & $\mathrm{T} 4$ & $\mathrm{~L} 1$ & 32 & 84 & 42 & 24 & 67 & 4 & 25 & 40 & 34 \\
\hline 7 & A & 20 & $\mathrm{~F}$ & $\mathrm{~T} 4$ & T9 & L2 & $\mathrm{T} 5$ & $\mathrm{~T} 12$ & 28 & 55 & 36 & 19 & 42 & 1 & 19 & 32 & 20 \\
\hline 8 & A & 16 & $\mathrm{M}$ & $\mathrm{T} 2$ & T9 & L3 & $\mathrm{T} 4$ & L1 & 19 & 53 & 27 & 8 & 12 & 13 & 5 & 16 & 14 \\
\hline 9 & A & 15 & M & $\mathrm{T} 3$ & T9 & L3 & $\mathrm{T} 2$ & $\mathrm{~T} 12$ & 48 & 59 & 23 & 49 & 33 & 13 & 39 & 40 & 16 \\
\hline 10 & A & 16 & M & $\mathrm{T} 2$ & T9 & L3 & $\mathrm{T} 4$ & L1 & 28 & 53 & 30 & 14 & 38 & 1 & 28 & 32 & 25 \\
\hline 11 & A & 18 & $\mathrm{~F}$ & $\mathrm{~T} 3$ & $\mathrm{~T} 8$ & L2 & $\mathrm{T} 4$ & $\mathrm{~T} 12$ & 21 & 50 & 28 & 14 & 28 & 9 & 18 & 19 & 7 \\
\hline 12 & A & 14 & $\mathrm{~F}$ & $\mathrm{~T} 2$ & T8 & L2 & $\mathrm{T} 4$ & L1 & 24 & 59 & 30 & 24 & 33 & 6 & 15 & 19 & 9 \\
\hline 13 & A & 14 & $\mathrm{~F}$ & $\mathrm{~T} 4$ & $\mathrm{~T} 10$ & L3 & T5 & $\mathrm{L} 1$ & 24 & 54 & 32 & 26 & 35 & 9 & 14 & 13 & 8 \\
\hline 14 & A & 36 & M & $\mathrm{T} 4$ & T9 & L3 & T5 & L1 & 34 & 64 & 23 & 25 & 24 & 7 & 30 & 24 & 1 \\
\hline 15 & A & 14 & $\mathrm{~F}$ & $\mathrm{~T} 3$ & T9 & L3 & $\mathrm{T} 4$ & L1 & 28 & 45 & 21 & 2 & 32 & 1 & 16 & 17 & 5 \\
\hline 16 & B & 16 & M & $\mathrm{T} 3$ & T9 & L3 & $\mathrm{T} 5$ & L1 & 38 & 67 & 48 & 23 & 41 & 15 & 16 & 30 & 34 \\
\hline 17 & B & 14 & $\mathrm{~F}$ & $\mathrm{~T} 3$ & T9 & L3 & $\mathrm{T} 4$ & L1 & 25 & 47 & 28 & 12 & 37 & 10 & 14 & 25 & 18 \\
\hline 18 & B & 16 & M & T3 & $\mathrm{T} 10$ & L3 & $\mathrm{T} 4$ & L1 & 24 & 54 & 33 & 10 & 21 & 0 & 25 & 27 & 23 \\
\hline 19 & B & 20 & $\mathrm{~F}$ & T3 & $\mathrm{T} 9$ & L3 & $\mathrm{T} 4$ & L1 & 32 & 58 & 32 & 16 & 34 & 13 & 22 & 29 & 20 \\
\hline 20 & B & 13 & $\mathrm{~F}$ & $\mathrm{~T} 2$ & $\mathrm{~T} 7$ & L2 & $\mathrm{T} 2$ & $\mathrm{~T} 12$ & 14 & 73 & 43 & 12 & 45 & 14 & 12 & 28 & 33 \\
\hline 21 & B & 13 & $\mathrm{~F}$ & T3 & $\mathrm{T} 10$ & L3 & $\mathrm{T} 3$ & L1 & 39 & 58 & 35 & 23 & 19 & 4 & 37 & 34 & 18 \\
\hline 22 & B & 16 & $\mathrm{~F}$ & $\mathrm{~T} 2$ & T9 & L3 & $\mathrm{T} 4$ & L1 & 26 & 77 & 54 & 11 & 27 & 14 & 23 & 26 & 21 \\
\hline 23 & B & 18 & $\mathrm{~F}$ & $\mathrm{~T} 3$ & $\mathrm{~T} 8$ & L2 & T6 & $\mathrm{T} 12$ & 36 & 52 & 43 & 12 & 17 & 7 & 21 & 26 & 27 \\
\hline 24 & B & 12 & $\mathrm{~F}$ & $\mathrm{~T} 2$ & $\mathrm{~T} 8$ & L3 & $\mathrm{T} 4$ & L1 & 46 & 62 & 32 & 35 & 40 & 4 & 29 & 31 & 21 \\
\hline 25 & B & 15 & $\mathrm{~F}$ & $\mathrm{~T} 3$ & T9 & L3 & T5 & L1 & 17 & 51 & 35 & 1 & 27 & 0 & 15 & 18 & 21 \\
\hline 26 & B & 16 & $\mathrm{~F}$ & $\mathrm{~T} 2$ & $\mathrm{~T} 8$ & L2 & $\mathrm{T} 4$ & L1 & 14 & 56 & 43 & 17 & 32 & 24 & 13 & 17 & 20 \\
\hline 27 & B & 18 & $\mathrm{~F}$ & $\mathrm{~T} 3$ & $\mathrm{~T} 8$ & L3 & $\mathrm{T} 4$ & $\mathrm{~T} 12$ & 26 & 60 & 34 & 10 & 26 & 17 & 15 & 26 & 11 \\
\hline 28 & B & 16 & $\mathrm{M}$ & $\mathrm{T} 2$ & $\mathrm{~T} 8$ & L3 & T3 & $\mathrm{T} 12$ & 31 & 72 & 46 & 7 & 43 & 22 & 16 & 38 & 46 \\
\hline 29 & B & 26 & $\mathrm{~F}$ & $\mathrm{~T} 3$ & T9 & L3 & $\mathrm{T} 4$ & L1 & 21 & 48 & 32 & 17 & 20 & 9 & 11 & 17 & 18 \\
\hline 30 & B & 14 & $\mathrm{~F}$ & $\mathrm{~T} 3$ & T8 & L2 & $\mathrm{T} 3$ & T12 & 44 & 66 & 36 & 35 & 58 & 3 & 21 & 15 & 12 \\
\hline 31 & $\mathrm{C}$ & 13 & $\mathrm{~F}$ & $\mathrm{~T} 3$ & T9 & L2 & $\mathrm{T} 2$ & $\mathrm{~T} 12$ & 46 & 79 & 60 & 31 & 41 & 0 & 27 & 26 & 31 \\
\hline 32 & $\mathrm{C}$ & 15 & $\mathrm{~F}$ & $\mathrm{~T} 3$ & $\mathrm{~T} 8$ & L3 & $\mathrm{T} 4$ & $\mathrm{~T} 12$ & 19 & 55 & 34 & 19 & 48 & 23 & 15 & 25 & 25 \\
\hline 33 & $\mathrm{C}$ & 13 & $\mathrm{~F}$ & $\mathrm{~T} 4$ & T9 & L2 & $\mathrm{T} 5$ & L1 & 21 & 59 & 45 & 12 & 39 & 11 & 15 & 26 & 29 \\
\hline 34 & $\mathrm{C}$ & 14 & $\mathrm{~F}$ & $\mathrm{~T} 2$ & $\mathrm{~T} 8$ & L2 & $\mathrm{T} 4$ & L1 & 29 & 91 & 67 & 21 & 59 & 31 & 22 & 59 & 53 \\
\hline 35 & $\mathrm{C}$ & 22 & $\mathrm{~F}$ & $\mathrm{~T} 2$ & $\mathrm{~T} 8$ & L2 & $\mathrm{T} 4$ & $\mathrm{~T} 12$ & 23 & 63 & 57 & 24 & 49 & 23 & 17 & 36 & 42 \\
\hline 36 & $\mathrm{C}$ & 24 & M & $\mathrm{T} 4$ & T9 & L2 & $\mathrm{T} 5$ & L1 & 26 & 57 & 42 & 24 & 50 & 7 & 30 & 51 & 33 \\
\hline 37 & $\mathrm{C}$ & 13 & $\mathrm{~F}$ & $\mathrm{~T} 3$ & T9 & L3 & T5 & L1 & 24 & 55 & 40 & 12 & 17 & 7 & 16 & 36 & 24 \\
\hline 38 & $\mathrm{C}$ & 20 & $\mathrm{~F}$ & $\mathrm{~T} 3$ & T9 & L3 & $\mathrm{T} 5$ & L1 & 25 & 50 & 42 & 21 & 43 & 21 & 17 & 25 & 35 \\
\hline
\end{tabular}

Values are given in degrees

$P T$ proximal thoracic curve, $M T$ main thoracic curve, $L$ lumbar curve, Instr instrumentation (The most proximal and distal vertebra that are fixated)

Table 3 Pre- and postoperative mean values

\begin{tabular}{|c|c|c|c|c|c|c|c|c|c|c|c|}
\hline & \multirow[t]{3}{*}{$N$} & \multicolumn{5}{|c|}{ Preoperative } & \multicolumn{5}{|c|}{ Postoperative } \\
\hline & & \multicolumn{3}{|l|}{ Thoracic } & \multicolumn{2}{|l|}{ Lumbar } & \multicolumn{3}{|l|}{ Thoracic } & \multicolumn{2}{|l|}{ Lumbar } \\
\hline & & PT & MT & AVT & $\mathrm{L}$ & AVT & PT & MT & AVT & $\mathrm{L}$ & AVT \\
\hline Entire group & 38 & $28(14-48)$ & $60(45-91)$ & $54(32-83)$ & $37(21-67)$ & $15(0-41)$ & $20(5-39)$ & $28(13-59)$ & $27(1-52)$ & $22(1-53)$ & $17(0-49)$ \\
\hline Lenke A & 15 & $28(17-48)$ & $57(45-84)$ & $58(40-74)$ & $30(21-42)$ & $7(0-13)$ & $20(5-39)$ & $26(13-40)$ & $28(11-52)$ & $14(1-34)$ & $8(0-20)$ \\
\hline Lenke B & 15 & $29(14-46)$ & $60(47-77)$ & $53(32-83)$ & $38(28-54)$ & $14(0-23)$ & $19(11-37)$ & $26(15-38)$ & $22(1-44)$ & $23(11-46)$ & $19(8-34)$ \\
\hline Lenke C & 8 & $27(19-46)$ & $64(50-91)$ & $53(41-70)$ & $48(34-67)$ & $30(22-41)$ & $20(15-30)$ & $36(25-59)$ & $30(1-52)$ & $34(24-53)$ & $29(15-49)$ \\
\hline
\end{tabular}

$\mathrm{PT}$ and MT in degrees and AVT in mm. Range is given between brackets

$P T$ proximal thoracic curve, $M T$ main thoracic curve, $L$ lumbar curve, $A V T$ apical vertebral translation 
Table 4 Pre- and postoperative sagittal alignment

\begin{tabular}{|c|c|c|c|c|c|c|c|c|c|c|}
\hline & \multicolumn{2}{|l|}{ T5-T12 } & \multicolumn{2}{|l|}{ T10-T12 } & \multicolumn{2}{|l|}{ T12-L2 } & \multicolumn{2}{|l|}{ L1-L5 } & \multicolumn{2}{|l|}{ L4-sacrum } \\
\hline & Pre & Post & Pre & Post & Pre & Post & Pre & Post & Pre & Post \\
\hline Enti & & & & & & & & & & 37 \\
\hline Lenke A & $24(8-68)$ & $24(13-33)$ & $6(1-18)$ & $6(1-11)$ & $6(0-16)$ & $5(1-9)$ & $42(24-72)$ & $43(24-58)$ & $37(27-56)$ & $34(11-54)$ \\
\hline Lenke B & $18(7-32)$ & $22(6-49)$ & $5(0-15)$ & $7(2-14)$ & $6(0-17)$ & $9(1-20)$ & $42(18-61)$ & $44(17-64)$ & $33(21-44)$ & $35(23-47)$ \\
\hline Lenke C & $27(6-62)$ & $27(9-67)$ & $10(1-18)$ & $9(2-19)$ & $5(1-10)$ & $8(2-15)$ & $51(39-62)$ & $46(32-68)$ & $40(28-53)$ & $47(37-60)$ \\
\hline
\end{tabular}

Mean values are given in degrees. Range is given between brackets

Table 5 Correlation of relative correction of main thoracic and lumbar curve, 1 year postoperatively

\begin{tabular}{lrrllll}
\hline & $N$ & M & \multicolumn{4}{c}{ Relative (\%) correction } \\
\cline { 3 - 7 } & & & MT & L & Corr & Sign \\
\hline Entire group & 38 & 12 & 54 & 44 & 0.590 & $\mathrm{P}<0.001$ \\
Lenke A & 15 & 13 & 56 & 54 & 0.698 & $\mathrm{P}=0.004$ \\
Lenke B & 15 & 13 & 57 & 41 & 0.526 & $\mathrm{P}=0.044$ \\
Lenke C & 8 & 12 & 44 & 29 & 0.492 & $\mathrm{P}=0.216$
\end{tabular}

$M$ number of months postoperatively, $M T$ relative (\%) correction of main thoracic curve $=[$ (correction of main thoracic curve in degrees/MT curve preoperatively) $\times 100 \%], L$ relative $(\%)$ correction of lumbar curve $=[$ (correction of lumbar curve in degrees/lumbar curve preoperatively) $\times 100 \%$, Corr correlation, Sign significance

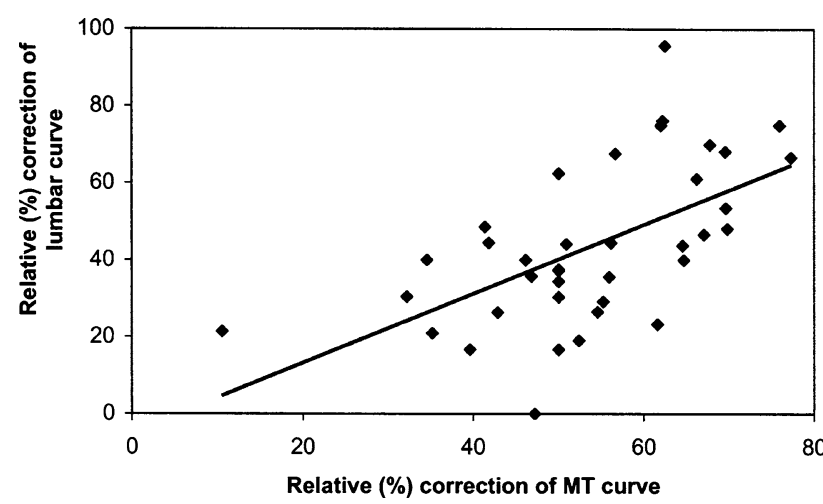

Fig. 1 Correlation of relative (\%) correction between the main thoracic (MT) curve and the lumbar curve $(R=0.590 ; P=0.001)$

\section{Discussion}

Idiopathic scoliosis is a complex deformity that deviates the trunk from its normal plane of symmetry and induces geometric changes of the spine in the three dimensions of space. Most authors assume that while a scoliosis develops, the vertebral column tries to find some form of equilibrium. Thus, in idiopathic adolescent thoracic scoliosis, a lumbar curve will develop to compensate for the deviation of the thoracic vertebrae and progression of the thoracic curve induces an increase of the lumbar curve. After selective thoracic fusion, the lumbar curve should spontaneously decrease $[3,6,7,10,16]$.

As expected, we achieved a significant correction of the lumbar curve following selective thoracic fusion as described in previous studies [3, 4, 6, 8, 11, 17]. In a previous study, we did not find a significant correlation between the correction of the thoracic and lumbar curve after fusion with Harrington instrumentation and sublaminar wiring [17]. Nowadays, new operation techniques are used and new classification systems are introduced. We assume, correlations will become more clear because of these.

In the present study, we used a third generation operation technique, with a posterior double rod instrumentation. A significant correlation was found between the relative (\%) corrections of the main thoracic and the lumbar curve 1 year postoperatively (Table 5) (Fig. 1). The correction of the lumbar curve seems to echo the correction obtained in the thoracic curve.

Despite the heterogeneity of final follow up, we found no significant difference between thoracic and lumbar correction 1-year postoperatively and at final follow up. The correlation of the relative corrections of the main thoracic and the lumbar curve was still present at final follow up $(R=0.530 ; P=0.001)$.

In literature, it is described that after selective correction and fusion of the main thoracic curve, the proximal thoracic curve decreased spontaneously as well [9]. The present study also shows a spontaneous correction of the proximal thoracic curve. Even more, a weak but significant correlation was present between the relative corrections of the main and the proximal thoracic curves $(R=0.419 ; P=0.009)$.

These correlations have not been described before. Using third generation instrumentation systems seems to give a better prediction of the correction of the different curves.

Lenke et al. [12] described a new classification system for scoliosis in which he divided the lumbar curve 
in three sub-groups (Table 1). We calculated correlations between the relative corrections of the main thoracic and lumbar curves for the lumbar modifier A, $\mathrm{B}$ and $\mathrm{C}$ groups (Table 5). For the subgroups, the correlation is strongest in the Lenke A group. For the Lenke B patients, the correlation is still present, but weaker. There is no significant correlation in the Lenke $\mathrm{C}$ group. Moreover, we determined balance in these patients and the results suggest, however not significant, that correction of trunkal shift depends on lumbar modifier. The lumbar modifier seems to be of predictable value for treatment outcome of the individual patient.

We presume that the three different types of lumbar curves have their own curve characteristics and an own correction mechanism. The Lenke $\mathrm{C}$ lumbar curve has the same real correction $\left(14^{\circ}\right)$ as type A $\left(15^{\circ}\right)$ and $\mathrm{B}$ $\left(15^{\circ}\right)$, but the relative correction decreases with the lumbar modifier (Lenke A 54\%, Lenke B 41\%, Lenke C $29 \%$ ). The lumbar curve seems to be composed of two curve-parts. An own structural curve, and a curve that is compensatory to the thoracic curve. After operation, the compensatory curve decreases because of decrease of the thoracic curve. However, the structural lumbar curve remains to exist, most clearly in Lenke C lumbar curves.

To better understand the correction of the lumbar curve we evaluated postoperative change of L4-tilt and the AVT [apical vertebral translation (Table 3)]. The lumbar apical vertebra is the vertebra which is most deviated from de central sacral line in the lumbar curve. AVT is the distance between this vertebra and the central sacral line. L4 tilt and AVT are smallest in Lenke A lumbar curves and largest in Lenke C lumbar curves. We were surprised that they both do not show a major decrease after operation (Fig. 2).

After operation, there was no significant improvement of L4 tilt and AVT for the whole group as for the three subgroups. This suggests that the lumbar correction had to occur proximal of the AVT. We conclude that the correction of the lumbar curve occurs mainly in the upper segment of the curve, above the apical vertebra. Figure 3 clearly shows the correction of the lumbar curve, which occurs mainly in the upper lumbar curve. Other investigators $[2,13,14,17]$ also described that the lumbosacral portion of the lumbar curve did not change after thoracic correction in idiopathic scoliosis. Recently we described the same phenomenon in patients with Scheuermann kyphosis. Lumbar hyperlordosis decreased spontaneously after surgical correction of the thoracic hyperkyphosis. The main lumbar correction was obtained in the upper lumbar segment [5].

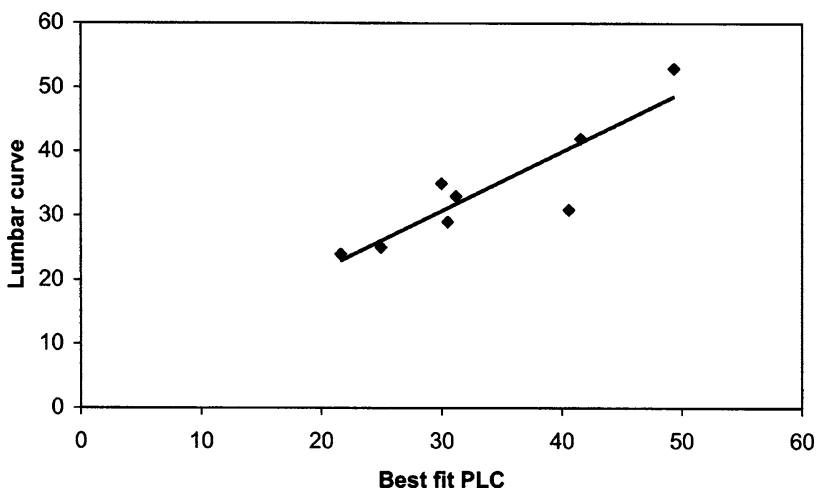

Fig. 2 Correlation between the predicted postoperative standing lumbar Cobb angle (PLC) and the postoperative lumbar curve in the Lenke $C$ group $(R=0.887 ; P=0.003)$
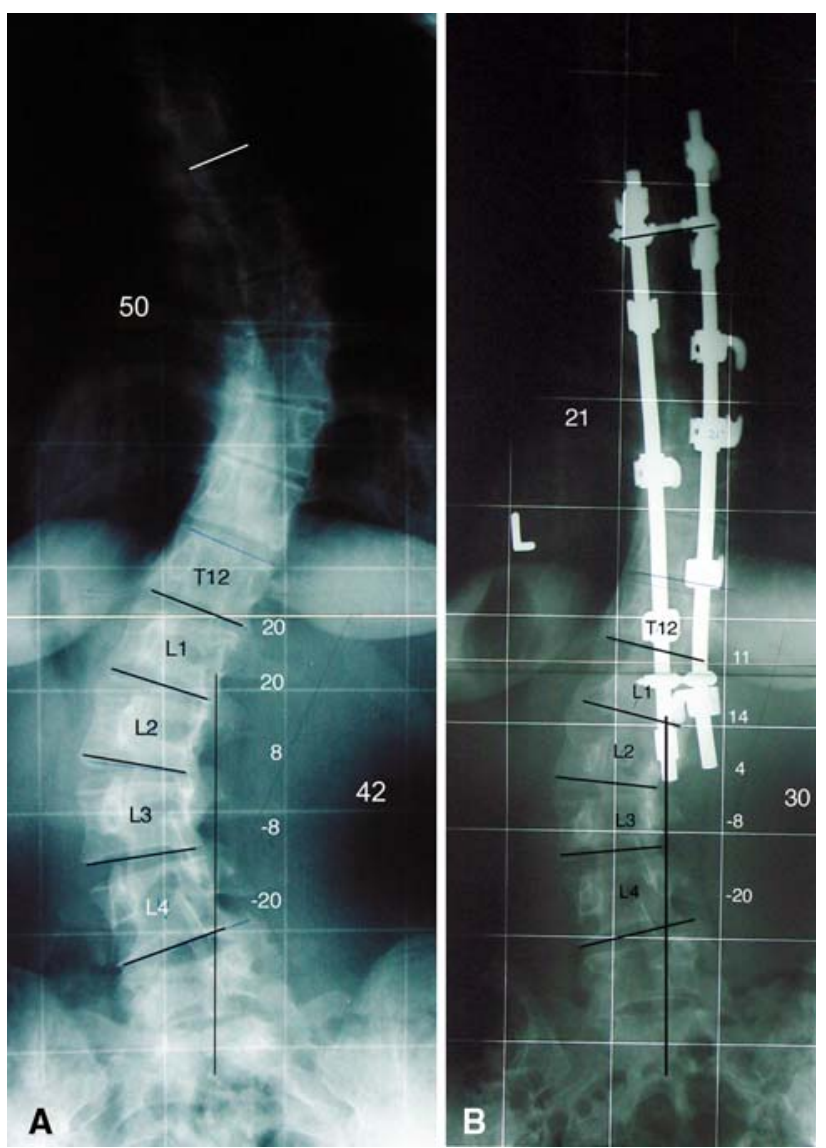

Fig. 3 Preoperative (a) and 2-year postoperative (b) frontal radiograph of one of the female patients, age 20, with lumbar modifier C. Values for main thoracic curve and lumbar curve are given in degrees. In the sagittal plane, pre- and postoperative kyphosis were $24^{\circ}$ and $28^{\circ}$, respectively. Pre- and postoperative lordosis were $51^{\circ}$ and $48^{\circ}$, respectively. In the frontal plane, considerable amount of correction of both curves is achieved. It is clearly seen that the lumbar curve corrects especially in the upper part of the lumbar curve 
This means that in both the frontal and sagittal plane, the distal lumbar curve is less flexible. It seems that the distal lumbar curve is not influenced by changes in other parts of the spine.

In literature, Mason et al. [14] proposed a formula to estimate the magnitude of the postoperative uninstrumented lumbar curve after correction of the right thoracic curve in idiopathic scoliosis. Their hypothesis was that all correction in the lumbar curve occurs by changing the angle of the upper segment of the lumbar Cobb angle while the lower segment of the lumbar Cobb angle remains in the same position. Their best-fit formula for the predicted postoperative follow-up standing lumbar Cobb angle $(\mathrm{PLC})$ is: $\mathrm{PLC}=(\mathrm{LC} \times 0.738)+$ $($ TC $\times-0.075)+($ BTC $\times 0.250)-8.030($ Table 6$)$.

If we calculate the predicted lumbar Cobb angle for our patients and we compare this with our postoperative measurements using Pearson correlation analyses we find a highly significant correlation for the Lenke $\mathrm{C}$ group ( $R=0.887 ; P=0.003$ ) (Fig. 2). Moreover, the paired samples $t$-test shows no significant difference between predicted and real postoperative curve (mean difference $0.29^{\circ} ; P=0.862$ ). For the Lenke A and $\mathrm{B}$ groups, the correlation between PLC and the real values is less significant.

For Lenke $\mathrm{C}$, the relative corrections of the main thoracic and lumbar curve do not correlate. However, for these patients, this formula seems to be of predictable value for the correction of the curve. This can be of great influence in the preoperative estimation of lumbar curve correction. Therefore it can assist in the decision whether to fuse the lumbar curve or not in patients with a Lenke C lumbar curve.

We mentioned before that the three different types of lumbar curves presumably have their own curve characteristics and an own correction mechanism. All lumbar curve types have the same real correction but not the same relative correction. The lumbar curve exist of an own structural part and a flexible compensatory part. The flexible part seems to be located in the upper part of the lumbar lordosis. This should guide us in the management of idiopathic scoliosis.

Table 6 Explanation of predicted postoperative standing lumbar Cobb angle (PLC) as described by Mason et al. [14]

$\mathrm{PLC}=(\mathrm{LC} \times 0.738)+(\mathrm{TC} \times-0.075)+(\mathrm{BTC} \times 0.250)-8.030$

PLC: predicted postoperative lumbar Cobb angle

LC: preoperative lumbar Cobb angle

TC: preoperative thoracic Cobb angle

BTC: preoperative bending thoracic Cobb angle
Our results suggest that if a thoracic scoliosis with a Lenke B or C lumbar curve are operated on, the instrumentation should not be extended into the lumbar curve, fixating the upper part of the lumbar curve. Postoperative, the flexible part of the lumbar spine would be fixated with loss of compensation mechanism with the risk of an unbalanced spine above the less flexible distal lumbar curve.

As described, a spontaneous correction of about $15^{\circ}$ is to be expected in the lumbar curve, independent of thoracic correction and lumbar curve type. Therefore it is important to be careful in correcting the thoracic spine. If one tries to achieve a maximal correction of the thoracic spine during surgery, the lumbar curve will probably fail in compensating this correction. This will result in an unbalanced spine, especially in type B and C lumbar curves.

It seems to be important to get more insight in the biodynamic mechanism of balance in the scoliotic spine. We can use this to achieve a better understanding of the construction of this deformity. This may lead to the development and testing of new treatment strategies.

\section{Conclusions}

1. With the use of third generation operation techniques, a highly significant correlation is present between the relative (\%) corrections of the main thoracic curve and the lumbar curve after selective thoracic fusion in idiopathic scoliosis. This may lead to a predictable correction of the lumbar curve.

2. The recently introduced new classification system seems to be of predictable value for the spontaneous correction of the lumbar curve after selective thoracic fusion in idiopathic scoliosis. Depending on the lumbar curve type, a different technique for predicting the outcome of the individual patient can be used.

3. Decrease of the lumbar curve after thoracic correction and fusion occurs mainly in the upper segments of the lumbar spine. The distal lumbar curve seems to be more rigid.

4. Our results suggest that thoracic correction should not be maximal but adjusted to the individual patient. The amount of correction in thoracic scoliosis with a lumbar modifier $\mathrm{B}$ and $\mathrm{C}$ depends on the flexibility of the upper part of the lumbar curve and the instrumentation should not be extended into the upper lumbar curve compromising the spontaneous lumbar curve correction. 


\section{References}

1. Bridwell KH (1994) Surgical treatment of adolescent idiopathic scoliosis: the basics and the controversies. Spine 19:1095-1100

2. Edwards CC, Lenke LG, Peelle M, Side B, Rinella A, Bridwell KH (2004) Selective thoracic fusion for adolescent idiopathic scoliosis with $\mathrm{C}$ modifier lumbar curves: 2- to 16year radiographic and clinical results. Spine 29:536-546

3. Frez R, Cheng JC, Wong EM (2000) Longitudinal changes in trunkal balance after selective fusion of King II curves in adolescent idiopathic scoliosis. Spine 25:1352-1359

4. Hosman AJ, Slot GH, Beijneveld WJ et al (1996) Correction of idiopathic scoliosis using the $\mathrm{H}$-frame system. Eur Spine $\mathrm{J}$ 5:172-177

5. Jansen RC, Van Rhijn LW, Van Ooij A (2006) Predictable correction of the unfused lumbar lordosis after thoracic correction and fusion in Scheuermann kyphosis. Spine 31(11):1227-1231

6. Kalen V, Conklin M (1990) The behavior of the unfused lumbar curve following selective thoracic fusion for idiopathic scoliosis. Spine 15:271-274

7. King HA, Moe JH, Bradford DS et al (1983) The selection of fusion levels in thoracic idiopathic scoliosis. J Bone Joint Surg Am 65:1302-1313

8. Knapp DR, Price CT, Jones ET et al (1992) Choosing fusion levels in progressive thoracal idiopathic scoliosis. Spine 17:1159-1165

9. Kuklo TR, Lenke LG, Won DS et al (2001) Spontaneous proximal thoracic curve correction after isolated fusion of the main thoracic curve in adolescent idiopahtic scoliosis. Spine 26:1966-1975

10. Large DF, Doig WG, Dickens DR et al (1991) Surgical treatment of double major scoliosis. Improvement of the lumbar curve after fusion of the thoracic curve. J Bone Joint Surg Br 73:121-124

11. Lenke LG, Betz RR, Bridwell KH et al (1999) Spontaneous lumbar curve coronal correction after selective anterior or posterior thoracic fusion in adolescent idiopathic scoliosis. Spine 24:1663-1671

12. Lenke LG, Betz RR, Harms J et al (2001) Adolescent idiopathic scoliosis: a new classification to determine extent of spinal arthrodesis. J Bone Joint Surg Am 83:1169-1181

13. Mason DE, Carrango P (1991) Spinal decompensation in Cotrel-Dubousset instrumentation. Spine 16(suppl 8):S394S403

14. Mason DE, Schindler A, King N (1998) Estimation of the lumbar curve magnitude with correction of the right thoracic curve in idiopathic scoliosis. J Pediatr Orthop 18:602-605

15. McCall RE, Bronson W (1992) Criteria for selective fusion in idiopathic scoliosis using Cotrel-Dubousset instrumentation. J Pediatr Orthop 12:475-479

16. Moe JH (1977) The classic. A critical analysis of methods of fusion for scoliosis an evaluation in two hundred and sixty-six patients. Clin Orthop 126:4-16

17. Van Rhijn LW, Plasmans CM, Veraart BE (2002) No relationship exists between the correction of the thoracic and the lumbar curves after selective thoracic fusion for adolescent idiopathic scoliosis King type II. Eur Spine J 11:550-555 